Gabriele Nase, Paul Johannes Helm, Tomohiro Oguchi, L. N. G. Nilsson, L. Lannfelt, Ole Petter Ottersen and Reidun Torp. "Local impact of perivascular plaques on cerebral blood flow dynamics in a transgenic mouse model of Alzheimer's disease." Proceedings of SPIE, vol. 6860, 2008.

(c) Copyright 2008 Society of Photo-Optical Instrumentation Engineers. One print or electronic copy may be made for personal use only. Systematic reproduction and distribution, duplication of any material in this paper for a fee or for commercial purposes, or modification of the content of the paper are prohibited.

http://dx.doi.org/10.1117/12.762739

Published in DUO with permission from spie.org

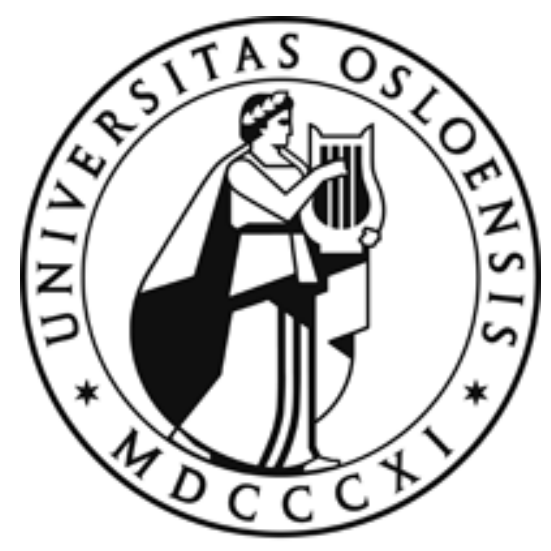




\title{
Local impact of perivascular plaques on cerebral blood flow dynamics in a transgenic mouse model of Alzheimer's disease
}

\author{
Gabriele Nase $^{1}$, P. Johannes Helm ${ }^{*}, 1$, Tomohiro Oguchi ${ }^{1}$, Lars N. G. Nilsson ${ }^{2}$, Lars Lannfelt ${ }^{2}$, Ole P. \\ Ottersen $^{1}$, Reidun Torp ${ }^{1}$ \\ ${ }^{1}$ Centre for Molecular Biology and Neuroscience and University of Oslo, Dept. of Anatomy, Oslo, \\ Norway \\ ${ }^{2}$ Department of Public Health / Molecular Geriatrics, Rudbeck Laboratory, Uppsala University, \\ Uppsala, Sweden
}

\begin{abstract}
Cerebrovascular pathology is closely coupled to cognitive function decline, as indicated by numerous studies at the system level. To better understand the mechanisms of this cognitive decline it is important to resolve how pathological changes in the vasculature - such as perivascular plaques - affect local cerebral blood flow dynamics. This issue is ideally studied in the intact brain at very high spatial resolution.

Here, we describe initial results obtained by an approach based on in vivo observation by multi-photon microscopy of vascular plaques and local blood flow measurements in a transgenic mouse model engineered to express the human amyloid precursor protein with the Swedish and Arctic mutations. These mice exhibit a striking abundance of perivascular plaques in the cerebral cortex and are well suited to investigate vascular pathology in Alzheimer's disease.
\end{abstract}

Keywords: MPLSM, plaque, amyloid, Alzheimer's, in vivo, perivascular, blood flow, mouse

\section{INTRODUCTION}

Cerebrovascular pathology is closely coupled to cognitive dysfunction, as indicated by numerous studies at system level ${ }^{1}$. Cerebral amyloid angiopathy (CAA) is a term used to describe the pathological changes occurring in cerebral blood vessels, resulting from deposition of amyloid proteins ${ }^{2}$. The term "amyloid" describes highly insoluble fibrils consisting of proteins rich in beta-pleated sheet secondary structure. Many amyloidogenic proteins are known and several staining methods are commonly used, such as dye substances named "Thioflavin S" or "Thioflavin T" (substances "T 1892" or "T 3516" Sigma-Aldrich Corporation, St. Louis, MO, USA) or "Methoxy-X04"”. These staining methods are specific for amyloid, as they have affinity for the beta-sheet content of amyloid. In addition, there are several commercially available antibodies that recognize the amyloidogenic protein and that distinguish between various fibrillar forms composed of $A \beta$ and nonfibrillar $A \beta$. The severity of CAA depends on the deposition of $A \beta$. Diffuse periarterial deposits lacking a well defined border are common around capillaries and arterioles. The deposits are often in continuity with $A \beta$ deposits in the adjacent neuropil and leave the endothelial cells intact. With increasing severity, $A \beta$ infiltrates all layers of the vessel wall and disrupts the vascular architecture ${ }^{4}$.

The local impact that pathological changes in the vasculature, such as perivascular plaques, exert on cerebral blood flow dynamics, and ultimately, on neural function is poorly understood and is ideally studied in the intact brain at very high spatial resolution. Here, we describe initial results obtained by an approach which uses in vivo observation of vascular $\mathrm{A} \beta$ deposits and local blood flow measurements in a transgenic Alzheimer mouse model characterized by a striking abundance of both intracortical and perivascular plaques.

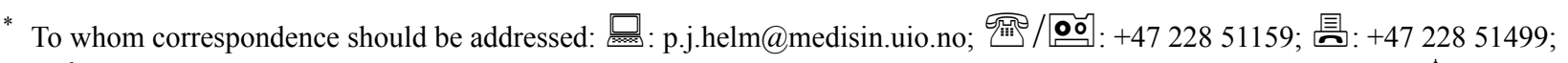
: P. Johannes Helm, University of Oslo, Dept. of Anatomy, P.O. Box 1105 - Blindern, NO-0317 Oslo, Norway; http://www.cmbn.no/group-ottersen.html
} 


\section{MATERIALS AND METHODS}

\subsection{Animals}

As a model for Alzheimer's disease (AD) we utilized 12-16 months old mice engineered to express the human amyloid precursor protein ${ }^{5}$ with the Swedish and Arctic mutations (APPArcSwe), displaying parenchymal and cerebrovascular amyloid deposits.. Young (3-6 months) and aged (around 16 months) C57BL/6 mice (Jackson Labs) served as control animals for optimizing staining protocols. In total, 13 animals of either sex were used. Surgery and preparation was performed as described earlier ${ }^{6}$. All experimental protocols were approved by the Institutional Animal Care and Use Committee and conform to the national guidelines for the care and use of animals. The number of animals used was kept at a minimum.

\subsection{In vivo imaging, data acquisition and analysis}

For in vivo imaging, a customized two-photon platform for in vivo imaging in mice as described earlier ${ }^{6}$ was used. Briefly, a model "FV300" laser scanning unit was mounted on a model BX51WI microscope (both Olympus Corp. Tokyo, Japan), and the light detecting photomultiplier tube, model R7683, a high efficiency OEM version of model R3896, was mounted in model C9143 Peltier cooling box equipped with a model C9145 DP-Type socket (all by Hamamatsu Photonics KK, Hamamatsu City, Japan) including a Cockroft-Walton style high DC voltqage source. The laser system was a model "Verdi 5W" / "Mira 900F" Nd:YVO / Ti:Sap unit (Coherent, Inc., Santa Clara, CA, USA). The beam of the Ti:Sap laser was controlled by means of a model "LM 0202P 5W IR / LIV8" Electro Optic Modulator system (Gsaenger / Linos, Planegg, FRG). The software package "Fluoview w/ O3D w/ TIEMPO, vs. 5.0 Build 107" (Olympus Corp. Tokyo, Japan) was used to control the scanning procedures (frame scans and line scans).

Plaques had been stained with Thioflavine S or Thiofalvine T, applied to the exposed cortex, or Methoxy-X04, injected subcutaneously $24 \mathrm{~h}$ in advance. For blood flow measurements, the blood plasma was labeled by intravenous injection of dextrane-coupled fluorescent dyes (Tetramethylrhodamineisothyocyanate - abbreviated "TRITC" - , dextran 3000MW, substance "D-3307", Invitrogen / Molecular Probes, Eugene, OR, USA). Subsequently, dynamic intensity measurements were performed by line scanning and 3D image stacks were acquired. Visualization of plaques around blood vessels was done at $860 \mathrm{~nm}$ excitation. This wavelength is suitable for both, Thioflavine $\mathrm{S}$ or T or Methoxy excitation and TRITC excitation. Fluorescence was detected via model "HQ 460/50M-2p" (Methoxy-04 and Thioflavins) or "E680sp-2p" (D3307) fluorescence filters (Chroma Technology Corp, Rockingham, VT, USA). The transmission band of the former filter is from $435 \mathrm{~nm}$ to $485 \mathrm{~nm}$, the latter is a broad band short pass filter transmitting the visible part of the spectrum and effectively blocking any wavelengths emitted by the Ti:Sap laser. Images at the different spectra were recorded consecutively and treated as individual channels during analysis. Image analysis, including 3D reconstructions and analysis of line scan data was performed off-line by means of software packages "Imaris, version 6.0" (Bitplane AG, Zürich, Switzerland), "ImageJ" 7, home made software in C and UNIX shell scripts for the extraction of time series data from line scan images $(\mathrm{PJH})$, and software package "Origin" 7.5, OriginLab Corporation, Northampton, MA, USA, for the statistical analysis).

\subsection{Histology}

The animals used for in vivo imaging were transcardially perfused and processed for immunohistochemistry. In brief; we used $4 \%$ formaldehyde in sodium phosphate buffer. The brains were kept in the fixative overnight. Series of horizontal sections were cut on a Vibratome. The sections were immunohistochemically stained for amyloid using the polyclonal "A $\beta 42 "$ antibody $^{8}$ or "6E10" (Signet Laboratories Inc, MA, USA). Sections were developed with diaminobenzidine (DAB) substrate using the avidin-biotin horseradish peroxidase system (Vector Labs). 


\section{RESULTS}

\subsection{Amyloid plaque deposition}
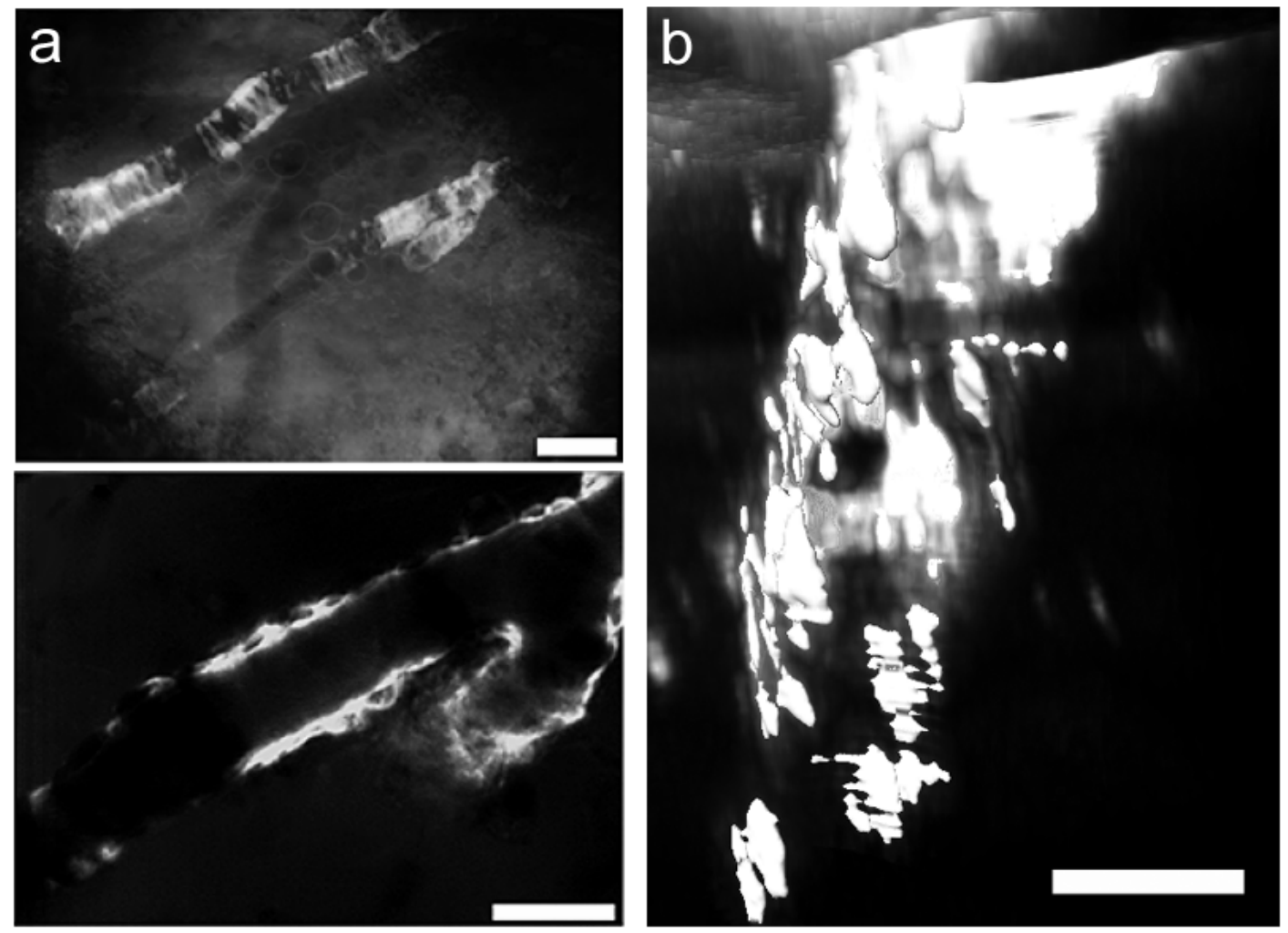

Figure 1. In vivo imaging of Thioflavin stained plaques in $\mathrm{AD}$ transgenic mice (a, scale bar 50 micron upper part and 20 micron lower part) versus wild type (b, scale bar 20 micron).

In vivo imaging revealed prominent staining representing perivascular plaques around some, but not all cerebral vessels in aged APPArcSwe animals, both using Thioflavine (Figure 1a) and Methoxy-X04 (Figure 2). Even in aged control animals, some vessels - presumably arterioles - exhibited striking Thioflavin positive material. The staining in control animals might represent arteriosclerotic plaques (Figure 1b). No Thioflavin staining was found in young control animals (not shown).

Immunocytochemistry confirms the findings obtained in vivo concerning the abundance and distribution of plaques (Figure 3). 


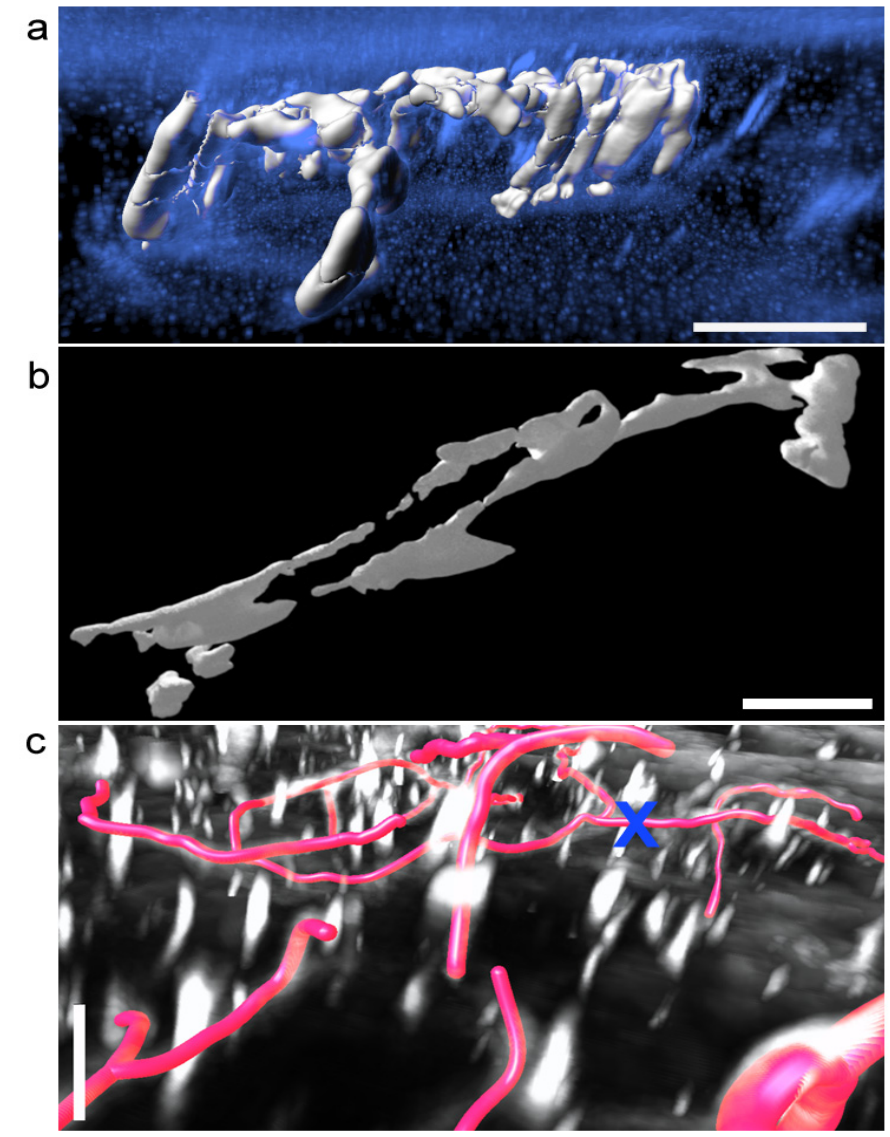

Figure 2. In vivo staining and 3D reconstruction of perivascular plaques and blood vessels in $\mathrm{AD}$ transgenic animals. Shown are examples of methoxyX04 positive structures surrounding big vessels (a, white structures, scale bar $50 \mu \mathrm{m})$, capillaries (b, scale bar $10 \mu \mathrm{m})$, and an example of reconstructed capillaries illustrating a site (blue cross) where blood flow measurements were taken (c, scale bar $50 \mu \mathrm{m}$ ).

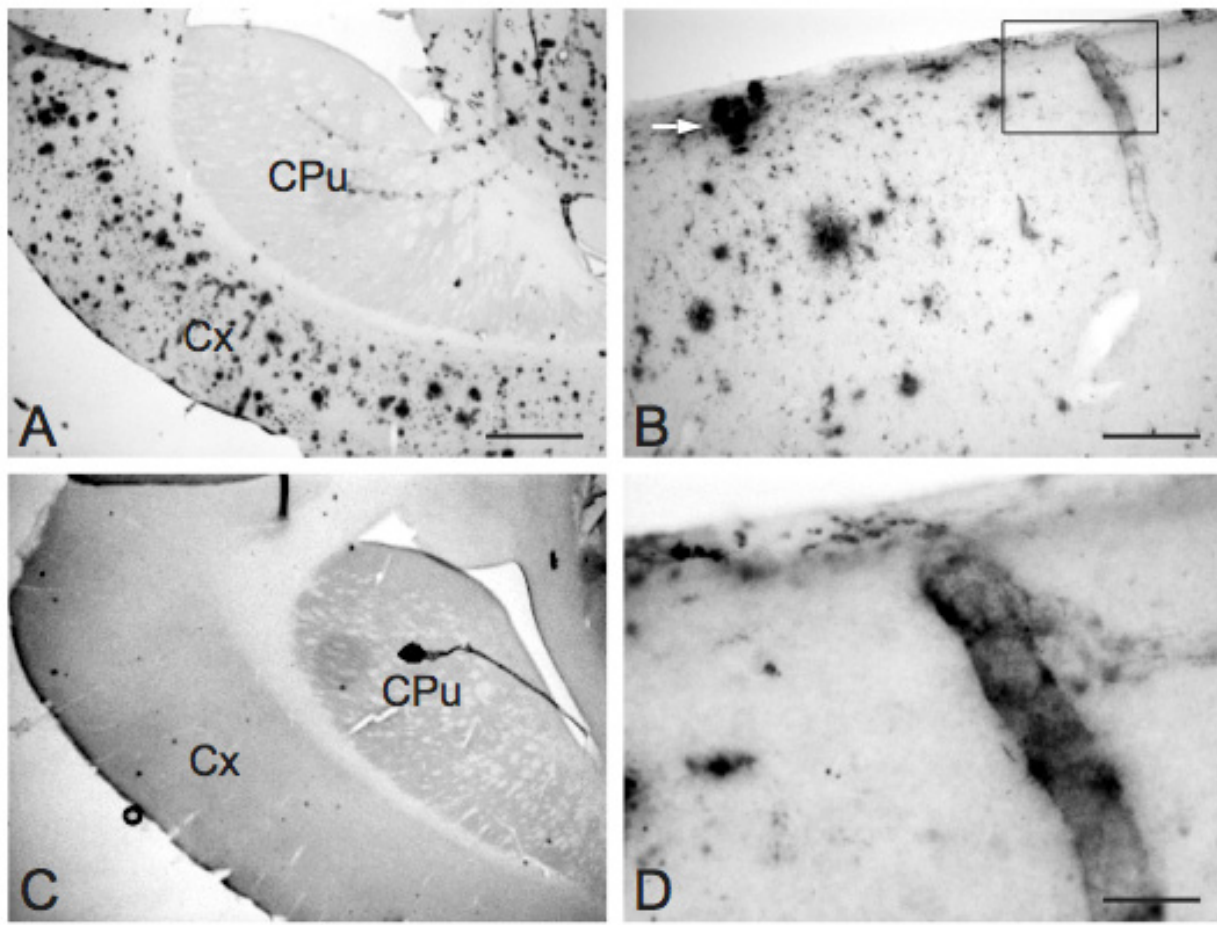

Figure 3. $A \beta$-ir plaque distribution in 16-monthold mice with the Artic and Swedish Alzheimer mutations. Panel A shows an overview of cortical distribution of $A \beta$ deposits. In panel $\mathrm{B}$, perivascular amyloid is observed close to the cortical surface. The framed vessel in B is magnified in panel D. An age-matched nontransgenic mouse is pictured in panel C. Cortex $(\mathrm{Cx}), \quad$ Caudate Putamen $(\mathrm{CPu})$. Scale bars: A $0.65 \mathrm{~mm}$, B $0.16 \mathrm{~mm}, \mathrm{D}$ $0.08 \mathrm{~mm}$ 


\subsection{Blood flow}

Initial analysis of blood flow measurements did not reveal any difference between AD transgenic animals $(n=4)$ and control animals $(\mathrm{n}=3)$ under base line activity, as shown in Figure 4. This preliminary data set comprises one measured site per animal.
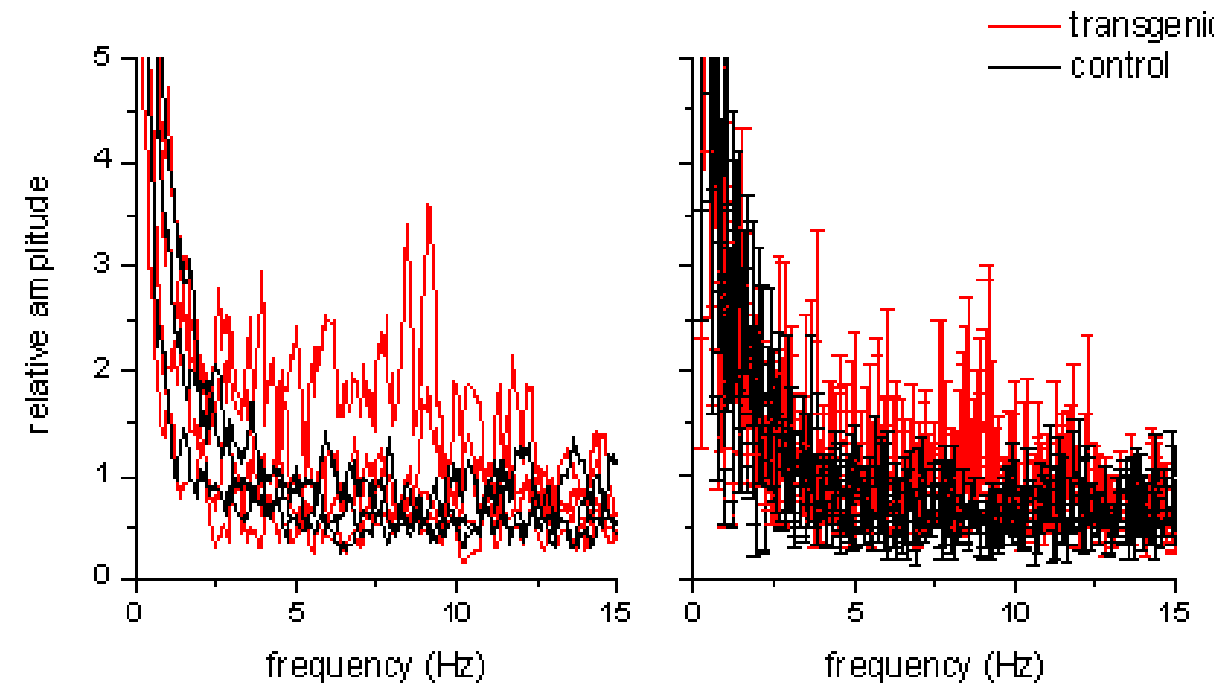

Figure 4. Local blood flow in $\mathrm{AD}$ transgenic versus control animals. FFT analysis of line scan data, individual measurements (left) and group average (right), (transgenic, $\quad \mathrm{n}=4$; control, $\mathrm{n}=3$; error bars indicate standard error of the mean).

\section{DISCUSSION}

The combination of in vivo multi-photon laser scanning microscopy (MPLSM) and fluorescent amyloid staining of perivascular plaques in aged APPArcSwe mice, as a model of Alzheimer's disease, permits analyses of the local impact of perivascular plaques on blood flow dynamics. In vivo application using methoxy-X04 yields a robust staining of plaques, including deposits on larger vessels and capillaries, as indicated by our comparison of in vivo and in vitro data. The animals displayed high frequency of extracellular plaques, including cerebrovascular amyloid deposits when examined with histochemical techniques. The Arctic mutation is unique among APP mutation, since it is located inside the $\mathrm{A} \beta$ domain and leads to $\mathrm{AD}$. It thereby alters the fibrillogenic characteristics of $\mathrm{A} \beta$ peptides resulting e.g. in more rapid $A \beta$ protofibril formation. Although CAA is seen in postmortem brain from mutation carriers, it is not considered as a model of CAA. However, we have chosen to work with this model since it produces $\mathrm{A} \beta$ protofibrils which constitute a form of large soluble $A \beta$ aggregates that is considered particularly toxic to the microenvironment. Mice with the Arctic mutation also develop intraneuronal $A \beta$ early in their life. Already within the first two month aggregates of $A \beta$ is observed. The relatively high load of perivascular amyloid could be assumed to affect local blood flow, by compressing the capillaries or reducing the flexibility of these. However, we were not able to observe differences in local blood flow between $\mathrm{AD}$ transgenic animals and control animals. Our data set, however, is preliminary and is currently extended to include analyses of activity dependent blood flow changes in response to sensory stimulation 


\section{ACKNOWLEDGEMENTS}

The multiphoton laser scan facilities and auxiliary infrastructure were financed jointly by the National Research Council and the University of Oslo Advanced Research Equipment Program, and by grants from Prof. Letten Fegersten Saugstad's Fund, the Jahre Foundation, and others awarded to the Laboratory of Molecular Neuroscience. Chemical substances used during the experiment were partly financed by the Norwegian chapter of CIVITAN International.

\section{REFERENCES}

1 Dietmar Rudolf Thal, Estifanos Ghebremedhin, Mario Orantes, and Otmar D. Wiestler, Vascular pathology in Alzheimer disease: correlation of cerebral amyloid angiopathy and arteriosclerosis/lipohyalinosis with cognitive decline, J. Neuropathol. Exp. Neurol. 62(12):1287-1301, 2003

2 G. G. Glenner and M.A. Murphy, Amyloidosis of the nervous system, J. Neurol. Sci. 94:1-28, 1989

3 William E. Klunk, Brian J. Bacskai, Chester A. Mathis, Stephen T. Kajdasz, Megan E. McLellan, Matthew P. Frosch, Manik L. Debnath, Daniel P. Holt, Yanming Wang, and Bradley T. Hyman, Imaging A $\beta$ plaques in living transgenic mice with multiphoton microscopy and methoxy-X04, a systemically administered Congo red derivative, J. Neuropathol. Exp. Neurol., 61:797-805, 2002

4 Johannes Attems, Sporadic cerebral amyloid angiopathy: pathology, clinical implications, and possible pathomechanisms Acta Neuropathol 110:345-359, 2005

5 Anna Lord, Hannu Kalimo, Chris Eckman, Xiao-Qun Zhang, Lars Lannfelt, and Lars N G. Nilsson, The Arctic Alzheimer mutation facilitates early intraneuronal $A \beta$ aggregation and senile plaque formation in transgenic mice, Neurobiology of Aging. 27(1):67-77, 2006

6 G. Nase, P.J. Helm, O.P.Ottersen, A multiphoton laser scanning microscope setup for transcranial in vivo brain imaging on mice, Review of Scientific Instruments 76:123702-1-123702-5, 2005

7 Abramoff, M.D., Magelhaes, P.J., Ram, S.J. "Image Processing with ImageJ", Biophotonics International, 11(7): 3642, 2004

8 Brian J Cummings and Carl W. Cotman, Image analysis of beta-amyloid load in Alzheimer disease and relation to dementia severity, Lancet 346:1524-1528, 1995 